Algebraic $\&$ Geometric Topology

Volume 1 (2001) 763-790

Published: 11 December 2001

\title{
Genus two 3-manifolds are built from handle number one pieces
}

\author{
ERIC SEDGWICK
}

\begin{abstract}
Let $M$ be a closed, irreducible, genus two 3-manifold, and $\mathcal{F}$ a maximal collection of pairwise disjoint, closed, orientable, incompressible surfaces embedded in $M$. Then each component manifold $M_{i}$ of $M-\mathcal{F}$ has handle number at most one, i.e. admits a Heegaard splitting obtained by attaching a single 1 -handle to one or two components of $\partial M_{i}$. This result also holds for a decomposition of $M$ along a maximal collection of incompressible tori.
\end{abstract}

AMS Classification 57M99

Keywords 3-manifold, Heegaard splitting, incompressible surface

\section{Introduction}

Throughout this paper, all surfaces and 3-manifolds will be taken to be compact and orientable. Suppose a 3-manifold $M$ contains an essential 2-sphere. The Haken lemma [4] tells us that each Heegaard surface for $M$ intersects some essential 2-sphere in a single essential circle (see also [5]). As a consequence of this and the uniqueness of prime decompositions of 3-manifolds, Heegaard genus is additive under connected sum,

$$
g\left(M_{1} \# \cdots \# M_{n}\right)=g\left(M_{1}\right)+\cdots+g\left(M_{n}\right),
$$

where $g(M)$ denotes the Heegaard genus of the manifold $M$.

How does Heegaard genus behave under decompositions of an irreducible manifold along incompressible surfaces? Clearly, we do not expect additivity of genus as before. Suppose that $M$ contains an embedded, incompressible surface $F$ that separates $M$ into two components $M_{1}$ and $M_{2}$. The genus of the two component manifolds must be greater than the genus of their boundary component, $g\left(M_{i}\right)>g(F), i=1,2$. This is particularly relevant in light of the examples of Eudave-Muñoz [2], tunnel number one knots whose exteriors contain incompressible surfaces of arbitrarily high genus. (An appropriate Dehn 
surgery on such a knot results in a closed genus two manifold with an arbitrarily high genus incompressible surface).

However, we can build a Heegaard splitting for $M$ from Heegaard splittings of the components $M_{1}$ and $M_{2}$. If done in an efficient manner, see for example [12], this yields an upper bound on the genus of $M$,

$$
g(M) \leq g\left(M_{1}\right)+g\left(M_{2}\right)-g(F) .
$$

Upper bounds on the genus of the component manifolds (lower bounds on $g(M))$ are more difficult, and not even possible without additional assumptions. Consider the examples of Kobayashi [8], knots whose tunnel numbers degenerate arbitrarily under connected sum (decomposition along an annulus). Again, an appropriate Dehn surgery will yield a closed manifold containing an incompressible torus, and after cutting along the torus, the component manifolds have genus arbitrarily higher than that of the closed manifold. In contrast, Schultens [13] has demonstrated that for tunnel numbers, this phenomenon cannot occur in the absence of additional incompressible surfaces. We are led to adding the assumption that the closed manifold should be cut along a maximal embedded collection of incompressible surfaces (a slightly weaker assumption will suffice, see the definition of a complete collection of surfaces in the next section).

While it is true that the spine of a Heegaard splitting for $M$ induces Heegaard splittings of the component manifolds, see Figure 3 and Section 4, the intersection between the Heegaard spine and incompressible surfaces could potentially be very complicated, and almost certainly depends on the genus of the incompressible surfaces. One approach to constructing upper bounds of the genus of the component manifolds is to bound the complexity of this intersection in terms of the genera of the incompressible surfaces and the Heegaard spine. This is the approach used by Johannson in [6].

In this paper, we adopt a different approach. Using ideas of Scharlemann and Thompson [11], we arrange the spine of the Heegaard splitting to intersect the collection of surfaces minimally. It is not hard to see that the induced Heegaard splitting of the component manifolds is weakly reducible. We then prove a generalization of a result of Casson and Gordon [1] to manifolds with boundary. (A similar theorem was proven by Lustig and Moriah [9].) A somewhat simplified version follows:

1.1 Theorem Let $M$ be an irreducible 3-manifold and $M=C_{1} \cup_{H} C_{2}$ a weakly reducible Heegaard splitting of $M$. Then either $M$ contains a closed, non-peripheral incompressible surface, or the splitting is not of minimal genus. 
This theorem allows us to make use of the assumption that we have taken the collection of surfaces to be complete. Additional refinements to this result show that for many of the component manifolds, the induced Heegaard splitting can be compressed to one that is induced by a single arc attached to the boundary.

1.2 Theorem Let $M$ be a closed, irreducible 3-manifold and $\mathcal{F}$ a complete collection of surfaces for $M$. If $\overline{M-N(\mathcal{F})}$ has $n$ component manifolds, then at least $n+2-g(M)$ of these components have handle number at most 1 .

We give definitions in the next section, but, note here that a complete collection of surfaces applies both to maximal collections of incompressible surfaces and maximal collections of incompressible tori. Handle number one means that the component manifold has a Heegaard splitting that is induced by drilling out a single arc, this is a generalization of tunnel number one and the concepts are identical when the manifold has a single boundary component. While it is possible that a component manifold has handle number 0 , this will occur only when $M$ fibers over the circle, or unnecessary parallel copies of some surface occur in the collection. Handle number 0 implies that the component is a compression body, in fact a product, since its boundary is incompressible. This component is either bounded by disjoint parallel copies of a surface, or there is a single surface cutting $M$ into a product, i.e., $M$ fibers over the circle.

Unfortunately, we are unable to draw conclusions about every component manifold unless the genus of $\Gamma$, hence $g(M)$, is 2 .

1.3 Corollary If $M$ is a closed, irreducible genus two 3-manifold and $\mathcal{F}$ is a complete collection of surfaces, then every component manifold of $\overline{M-N(\mathcal{F})}$ has handle number at most 1.

Although the component manifolds have high genus Heegaard splittings, the fact that they are handle number one means that this is due precisely to the fact that these manifolds have boundary with high genus, and are otherwise very simple in terms of Heegaard structure. The genus of a handle number one component manifold is bounded above by

$$
g\left(M_{i}\right) \leq g\left(\partial M_{i}\right)+1
$$

where $g\left(\partial M_{i}\right)$ is the sum of the genera of the components of $\partial M_{i}$. In some cases, this allows one to precisely compute the genus of the component manifolds. For example, when $g(M)=2$ and the complete collection $\mathcal{F}$ consists of a single separating surface $F$, we obtain the equality $g\left(M_{i}\right)=g(F)+1, i=1,2$. By contrast, in this case Johannson obtains a bound of $g\left(M_{1}\right)+g\left(M_{2}\right) \leq 2 g(F)+10$. 
Or, if $\mathcal{F}$ is a maximal embedded collection of tori, the single handle is either attached to one boundary component or is an arc joining two distinct torus boundary components. As a corollary, we obtain:

1.4 Corollary If $M$ is genus 2 , and $\mathcal{F}$ is a maximal collection of tori then every component of $\overline{M-N(\mathcal{F})}$ has genus 2 .

Kobayashi [7] has proven a much stronger result regarding torus decompositions of genus 2 manifolds.

Most of the techniques presented here apply without assumption on the genus of $M$. The exception is Proposition 6.3 whose hypothesis on handle number will not be consistently met when the genus of $M$ is greater than two. If the hypothesis on handle number can be removed, then a general upper bound on the handle numbers of the component manifolds is obtained. (It is likely that one must adopt the assumption that the collection $\mathcal{F}$ is in fact maximal). This would yield:

1.5 Conjecture Let $M$ be a closed, irreducible 3-manifold and $\mathcal{F}$ a maximal embedded collection of orientable, incompressible surfaces. If $M-N(\mathcal{F})$ has $n$ components then

$$
\sum_{1}^{n} h\left(M_{i}\right) \leq g(M)+n-2,
$$

where $h\left(M_{i}\right)$ denotes the handle number of the component manifold $M_{i}$.

\section{Preliminaries}

We give brief definitions of concepts related to Heegaard splittings, the reader is referred to [10] for a more thorough treatment. Let $S$ be a closed surface, $I=[-1,1]$. A compression body $C$ is a 3 -manifold obtained by attaching 2handles and 3-handles to $S \times I$, where no attachment is performed along $S \times\{1\}$. The boundary of a compression body is then viewed as having two parts, $\partial_{+} C$ and $\partial_{-} C$, where $\partial_{+} C=S \times\{1\}$ and $\partial_{-} C=\partial C-\partial_{+} C$. Alternatively, we may construct a compression body $C$ by attaching 1 -handles to $S \times I$ where all attachments are performed along $S \times\{1\}$. In this case, $\partial_{-} C=S \times\{-1\}$, $\partial_{+} C=\partial C-\partial_{-} C$, and the 1-handles are dual to the 2-handles of the former construction. In either construction, we adopt the convention that every 2 sphere boundary component of $\partial_{-} C$ is capped off with a ball. If $\partial_{-} C=\emptyset$ 
then $C$ is called a handlebody. Note that a handlebody can also be defined as a connected manifold with boundary that possesses a complete collection of compressing disks, a properly embedded collection of disks (the cores of the 2-handles) which cut the handlebody into a disjoint union of balls.

A Heegaard splitting is a decomposition of a (closed or bounded) 3-manifold, $M^{\prime}=C_{1} \cup_{S} C_{2}$, where $C_{1}$ and $C_{2}$ are compression bodies with their positive boundaries identified, $S=\partial_{+} C_{1}=\partial_{+} C_{2}$. In this case $S$ will be a closed surface embedded in $M^{\prime}$ and will be called a Heegaard surface for $M^{\prime}$. The genus of $M^{\prime}$ is

$$
g\left(M^{\prime}\right)=\min \left\{g(S) \mid S \text { is a Heegaard surface for } M^{\prime}\right\} .
$$

A Heegaard splitting will be called weakly reducible if there are non-empty properly embedded collections of compressing disks $\Delta_{1} \subset C_{1}$ and $\Delta_{2} \subset C_{2}$ so that $\partial D_{1} \cap \partial \Delta_{2}=\emptyset$ in the Heegaard surface $S$. If it exists, the collection $\Delta_{0}=\Delta_{1} \cup \Delta_{2}$ is called a weak reducing system for the Heegaard splitting.
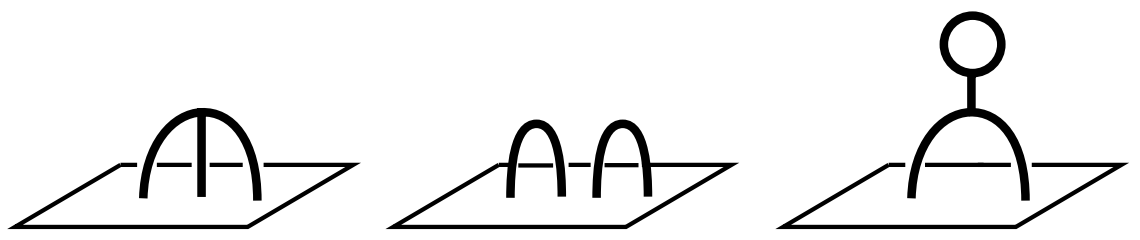

Figure 1: Graphs with handle number 2.

If $\Gamma$ is a graph then we will refer to the vertices of valence 1 , as the boundary of $\Gamma, \partial \Gamma$. A graph $\Gamma \subset M^{\prime}$ will be said to be properly embedded if it is embedded in $M$ and $\Gamma \cap \partial M^{\prime}=\partial \Gamma$. For a properly embedded graph $\Gamma \subset M^{\prime}$, we will define the genus of $\Gamma$ to be

$$
g(\Gamma)=\operatorname{rank} H_{1}(\Gamma)
$$

and define the handle number of $\Gamma$ to be

$$
h(\Gamma)=\operatorname{rank} H_{1}(\Gamma, \partial \Gamma) .
$$

Equivalently, the handle number is the number of edges that need to be removed from $\Gamma$ so that the resulting graph is empty or a collection of trees each attached to a boundary component of $M^{\prime}$ by a single vertex; or, $h(\Gamma)=-\chi(\Gamma)+|\partial \Gamma|=$ $g(\Gamma)+|\partial \Gamma|-|\Gamma|$. Some handle number two graphs are pictured in Figure 1.

Typically we will keep track of a Heegaard splitting via a properly embedded graph in the manifold. A Heegaard splitting of a closed manifold $M$ will necessarily consist of two handlebodies, and in this case, each of the handlebodies 
is isotopic to a regular neighborhood of a (non-unique) graph embedded in the handlebody, hence the manifold. Any such graph $\Gamma$, for either handlebody, will be called a spine of the Heegaard splitting. For bounded manifolds, Heegaard splittings come in two varieties, depending on whether or not one of the compression bodies is actually a handlebody. Correspondingly, there are two ways that a properly embedded graph can represent a Heegaard splitting of a bounded manifold. A tunnel system for $M^{\prime}$ is a properly embedded graph $\Gamma$ so that $\overline{M^{\prime}-N(\Gamma)}$ is a handlebody. The tunnel number of $M^{\prime}$ is

$$
t\left(M^{\prime}\right)=\min \left\{h(\Gamma) \mid \Gamma \text { is a tunnel system for } M^{\prime}\right\} .
$$

A handle system for $M^{\prime}$ is a properly embedded graph $\Gamma$ so that $\overline{M^{\prime}-N(\Gamma)}$ is a compression body $C$ and $\partial_{-} C \subset \partial M^{\prime}$. The handle number of $M^{\prime}$ is

$$
h\left(M^{\prime}\right)=\min \left\{h(\Gamma) \mid \Gamma \text { is a handle system for } M^{\prime}\right\} .
$$

In either case, if $\partial_{1} M^{\prime}$ denotes the boundary components of $M^{\prime}$ to which $\Gamma$ is attached, then $\partial N\left(\Gamma \cup \partial_{1} M^{\prime}\right)$ is a Heegaard surface for $M^{\prime}$.

Whenever a Heegaard splitting is represented by an embedded graph, whether a spine, tunnel system, or handle system, then we may perform slides of edges of the graph along other edges of the graph without changing the isotopy class of the Heegaard surface, see Figure 2. Such moves are called edge slides or handle slides. When working with tunnel or handle system, we may also slide handles along the boundary of the manifold without changing the Heegaard splitting.
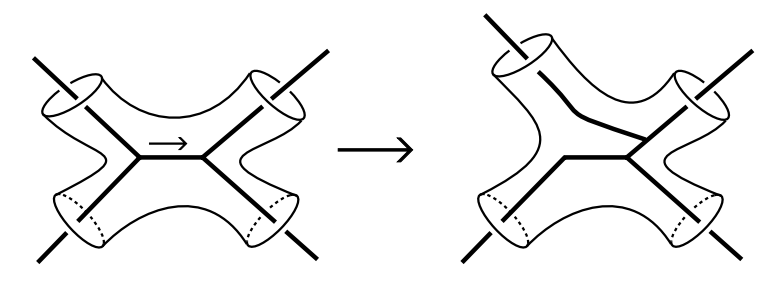

Figure 2: Edge slides do not change the Heegaard splitting.

In the case of a tunnel or handle system, $\Gamma$ will be slide-equivalent to a collection of $h(\Gamma)$ properly embedded arcs in $M^{\prime}$. So $t\left(M^{\prime}\right)$ and $h\left(M^{\prime}\right)$ should be thought of as the minimal number of arcs that need to be drilled out of $M^{\prime}$ so that the resulting manifold is a handlebody or compression body, respectively. The handle number is a strict generalization of the tunnel number and we have $h\left(M^{\prime}\right) \leq t\left(M^{\prime}\right)$. In general these quantities are different. For example the exterior of the Hopf link in $S^{3}$ is tunnel number one but handle number 0.

A bounded manifold $M^{\prime}$ will be said to be indecomposable if it contains no closed, orientable, non-peripheral incompressible surface whose genus is either 
less than or equal to the genus of a single boundary component of $M^{\prime}$ or strictly less than the sum of the genera of two distinct boundary components of $M^{\prime}$. Let $\mathcal{F}=F_{1} \cup F_{2} \cup \ldots, F_{k} \subset M$ be an embedded collection of closed orientable incompressible surfaces. A component manifold is a component of the manifold $\overline{M-N(\mathcal{F})}$. If $\mathcal{F}$ is an embedded collection of closed, orientable, incompressible surfaces and each of the component manifolds is indecomposable, then we say that $\mathcal{F}$ is a complete collection of surfaces.

Clearly a maximal embedded collection of orientable, incompressible surfaces is complete. However, this is not required for the collection be complete. For example, a maximal embedded collection of tori in an irreducible manifold is complete as each of the component manifolds is indecomposable (any additional surface would have to be genus 1).

\section{Proof of the Main Theorem}

In this section we will give an outline of the proof of the main theorem, Theorem 1.2. The proofs of several important lemmas will be deferred to later sections. Throughout, $M$ will denote a closed, orientable, irreducible 3 -manifold, $\Gamma$ will be the spine of an irreducible Heegaard splitting of $M$, and $\mathcal{F}$ will be a complete collection of incompressible surfaces.

Arranging $\Gamma$ to intersect the decomposition minimally Embed in $M$ two parallel copies of each of the incompressible surfaces in $\mathcal{F}$ and denote this collection by $2 \mathcal{F}$. If there are $k$ components of $\mathcal{F}, 2 \mathcal{F}$ decomposes $M$ into $n+k$ pieces, $k$ product manifolds $F_{j} \times I, j=1 . . k$ and $n$ component manifolds denoted $M_{i}, i=1 . . n, n<k$, identical to those obtained by cutting along $\mathcal{F}$. See Figure 3.

Suppose that $\Gamma$ is in general position with respect to $2 \mathcal{F}$ and that we have chosen $\Delta$ to be a complete collection of compressing disks for the complementary handlebody $\overline{M-N(\Gamma)}$. The complexity of $(\Gamma, \Delta)$ is an ordered triple $(\cdot, \cdot, \cdot)$ of the following quantities:

(1) $\sum h\left(\Gamma \cap M_{i}\right)=$ the sum of handle numbers of the intersection of the spine $\Gamma$ with each of the component manifolds $M_{i}$,

(2) $\sum h\left(\Gamma \cap\left(F_{j} \times I\right)\right)=$ the sum of the handle numbers of the intersection of the spine $\Gamma$ with each of the product manifolds $F_{j} \times I$,

(3) $|\Delta \cap 2 \mathcal{F}|=$ the number of components in the intersection of the disk collection $\Delta$ and the surfaces $2 \mathcal{F}$. 


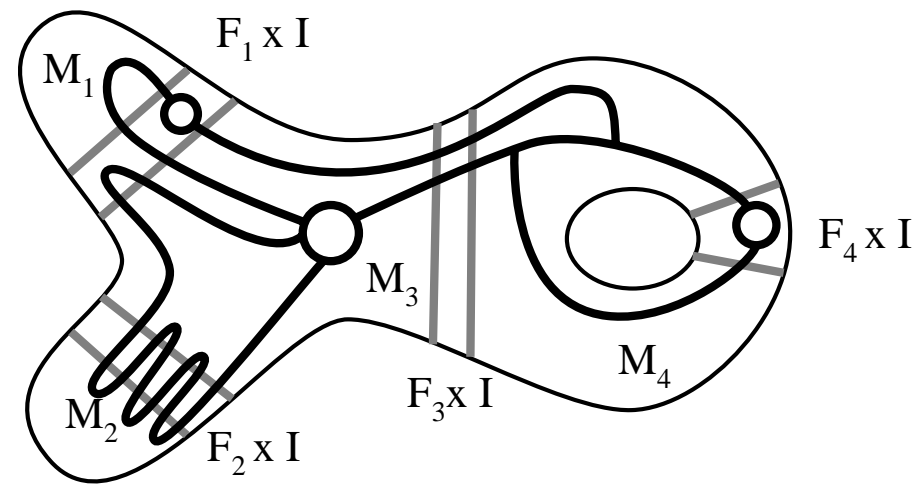

Figure 3: Letting $\Gamma$ intersect the decomposition minimally.

Isotoping or manipulating $\Gamma$ by edge-slides does not change the isotopy class of the Heegaard surface $\partial N(\Gamma)$, and we therefore consider such a spine to be equivalent to $\Gamma$. With no loss of generality, we will assume that a spine equivalent to $\Gamma$ and a complete collection of compressing disks $\Delta$ have been chosen to minimize complexity with respect to lexicographic ordering. Specific properties of the intersection $(\Gamma \cup \Delta) \cap 2 \mathcal{F}$ will be developed in Section 4; and are based on the arguments of Scharlemann and Thompson [11]. In particular we will prove:

3.1 Theorem $\Gamma \cap M^{\prime}$ is a tunnel system for each product or component manifold $M^{\prime}$.

Proof deferred to Section 4.

Ordering subdisks of $\Delta-2 \mathcal{F}$ By Lemma 4.3 we know that $\Delta-2 \mathcal{F}$ is a collection of disks. We will (non-uniquely) label these disks $d_{1}, \ldots, d_{m}$ according to the following rules:

(1) Label an outermost disk $d_{1}$,

(2) Assuming that the disks $d_{1}, \ldots, d_{l-1}$ have been labeled, give the label $d_{l}$ to a subdisk of $\Delta-2 \mathcal{F}$ that is outermost relative to the subdisks $d_{1}, \ldots, d_{l-1}$. See Figure 4 . 

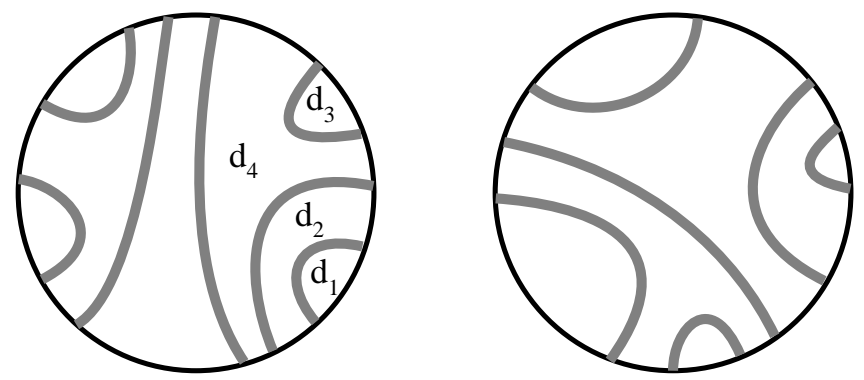

Figure 4: Labeling subdisks of $\Delta-2 \mathcal{F}$.

Note that each of the subdisks $d_{l}$ is embedded in some component or product manifold $M^{\prime}$. Moreover, it is a compressing disk for the handlebody that is the complement of the tunnel system induced by $\Gamma, \overline{M^{\prime}-N(\Gamma)}$.

Let $\left\{d_{i_{j}}\right\}$ be a subcollection of the disks $\Delta-2 \mathcal{F}$. The support of $\left\{d_{i_{j}}\right\}$, denoted $\operatorname{supp}\left(\left\{d_{i_{j}}\right\}\right)$, is the sub-graph of $\Gamma$ that is the spine of the handlebody obtained by maximally compressing $N(\Gamma)$ along compressing disks which are disjoint from $2 \mathcal{F}$ and disjoint from the boundary of $\left\{d_{i_{j}}\right\} \subset \partial N(\Gamma)$ and throwing away any components which do not meet $\left\{d_{i_{j}}\right\}$. For each component manifold $M_{i}$ let $j$ be the least $j$ so that $d_{j} \subset M_{i}$. The disk $D_{i}=d_{j}$ will be called the relatively outermost disk for $M_{i}$. The graph

$$
\Omega_{i}=\operatorname{supp}\left(D_{i}\right)
$$

will be called the relatively outermost graph for $M_{j}$.

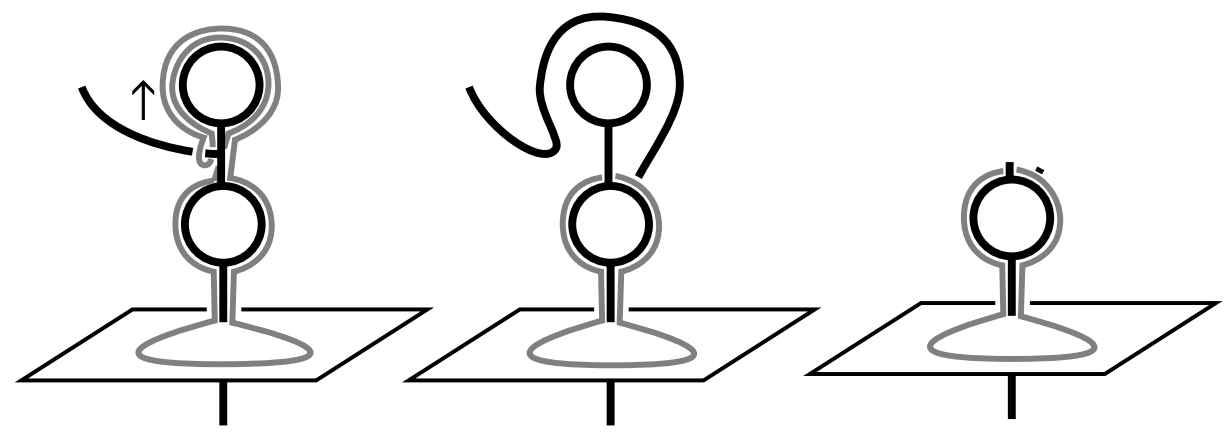

Figure 5: The support of a disk. 


\section{Remarks}

(1) In the definition of support, it may be necessary to perform handle slides of $\Gamma$ in the interior of some component manifolds in order to realize the maximal collection of compressing disks, see Figure 5.

(2) We have chosen a fixed numbering of the subdisks of $\Delta-2 \mathcal{F}$. Thus, the notions of the relatively outermost disk and the relatively outermost graph for a component manifold are well defined.

(3) We will consider the support of a relatively outermost disk $\Omega_{i}=\operatorname{supp}\left(D_{i}\right)$ to be a graph that is properly embedded in the component manifold $M_{i}$. We will consider the support of a collection of subdisks $\operatorname{supp}\left(\left\{d_{i_{j}}\right\}\right)$ to be graph that is embedded in $M$.

We can reconstruct the spine $\Gamma$ by building a sequence of graphs, each the support of a larger collection of ordered subdisks of $\Delta-2 \mathcal{F}$,

$$
\Gamma_{k}=\operatorname{supp}\left(\left\{d_{i}\right\}_{i=1 . . k}\right) .
$$

In particular,

$$
\Gamma_{m}=\Gamma .
$$

The relatively outermost graphs for each component manifold, $\Omega_{1}, \ldots, \Omega_{n}$, will be attached at some point in building $\Gamma$. Moreover, they are the support of the relatively outermost disks $D_{1}, \ldots, D_{n}$, and as we will see they are attached to the previous graph along all but at most one of their endpoints. This gives us a lower bound for the genus of $\Gamma$ in terms of the handle number of the outermost graphs $\Omega_{i}$.

3.2 Lemma Let $\Gamma$ be the spine of an irreducible Heegaard splitting. Then

$$
g(\Gamma) \geq \sum_{1}^{n} h\left(\Omega_{i}\right)-n+2 .
$$

Proof deferred until Section 5.

However, it is our aim to develop a lower bound for the genus of $\Gamma$ in terms of the handle numbers of the component manifolds, not just the handle numbers of the relatively outermost graphs $\Omega_{i}$. In a special case $\left(h\left(\Omega_{i}\right)=1\right)$ we will show that $\Omega_{i}$ is in fact a handle system and obtain the desired bound.

3.3 Proposition If $h\left(\Omega_{i}\right)=1$ then $\Omega_{i}$ is a handle system for $M_{i}$. In particular, $h\left(M_{i}\right) \leq 1$. 
Proof deferred to Section 6.

Remark The restriction $h\left(\Omega_{i}\right)=1$ in this proposition is what prevents us from making a more general statement connecting genus to the sum of handle numbers of the component manifolds. If $\Omega_{i}$ were always a handle system for the component manifold $M_{i}$ then we would obtain the more general inequality $g(\Gamma) \geq \sum h\left(M_{i}\right)-n+2$.

These lemma and proposition prove the main theorem. Let $j \leq n$ be the number of components $M_{i}$ which have $h\left(M_{i}\right)>1$. By Proposition 3.3, each of the corresponding outer handle systems $\Omega_{i}$ has $h\left(\Omega_{i}\right)>1$. By Lemma 5.1 we have

$$
g(\Gamma)-2 \geq \sum h\left(\Omega_{i}\right)-n \geq n+j-n \geq j .
$$

Therefore, the number of component manifolds with handle number one is at least

$$
n-(g(\Gamma)-2)=n+2-g(\Gamma) .
$$

\section{Properties of the Minimal Intersection between $2 \mathcal{F}$ and $\Gamma$}

This section is devoted to developing the properties of the minimal intersection between the Heegaard complex $\Gamma \cup \Delta$ and the incompressible surfaces $2 \mathcal{F}$. Many of these properties were either specified in the work of Scharlemann and Thompson [11], or follow from the same methods. They are included here, both for the sake of completeness, and due to the fact that the definition of minimality used here differs from that in [11]. We also apply these properties to characterize the support of outermost and relatively outermost disks.

Throughout this section, we assume that the spine $\Gamma$ of the irreducible Heegaard splitting and compressing disks $\Delta$ for its complement have been chosen to intersect the surfaces $2 \mathcal{F}$ minimally, as defined in the previous section. However, it is not necessary to place any restrictions on the surface collection $\mathcal{F}$.

First we will demonstrate that $\Gamma$ induces Heegaard splittings of each of the component and product manifolds.

4.1 Lemma Let $F$ be a component of $2 \mathcal{F}$. Then the punctured surface $F^{\prime}=\overline{F-N(\Gamma)}$ is incompressible in the handlebody $\overline{M-N(\Gamma)}$. 
Proof If some component of the punctured surface were compressible, then there would be a compressing disk $D$ for $F^{\prime}$, a perhaps distinct component of the punctured surface, embedded in some component or product manifold $M^{\prime}$. The boundary of $D$ bounds a disk $D^{\prime}$ in $F$. As $M$ is irreducible, $D$ and $D^{\prime}$ cobound a ball $B$, and $B$ must be contained in $M^{\prime}$, for otherwise the incompressible surface $F$ would lie in the ball $B$. We can therefore isotope $F$ through $B$, thereby pushing a portion of $\Gamma \cap M^{\prime}$ into an adjacent product or component manifold. See Figure 6 . Since $\Gamma$ is the spine of an irreducible splitting, by a theorem of Frohman [3], $B \cap \Gamma$ does not contain any loops of $\Gamma$, it is merely a collection of trees. As there is no loop of $\Gamma$ in the ball, this move does not raise the induced handle number of the adjacent manifold, while it does reduce the handle number of $\Gamma \cap M^{\prime}$. This contradicts the minimality of the intersection.

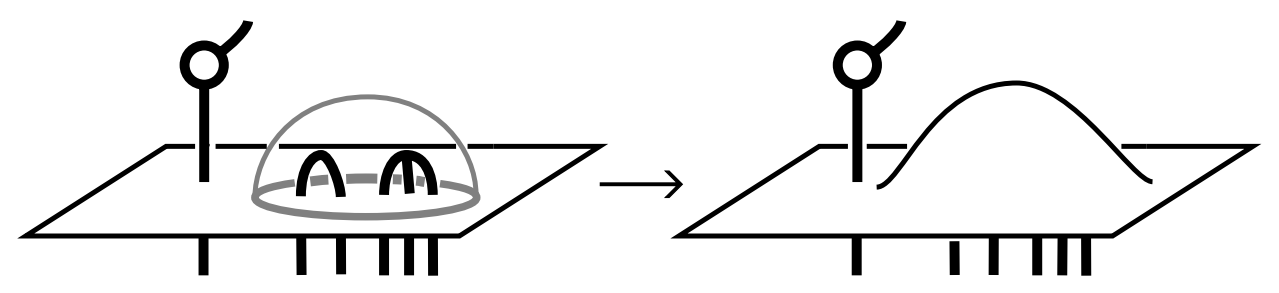

Figure 6: If $F^{\prime}$ is compressible the intersection is not minimal.

4.2 Theorem $\Gamma \cap M^{\prime}$ is a tunnel system for each product or component manifold $M^{\prime}$.

Proof The manifold $\overline{M^{\prime}-N(\Gamma)}$ is a component of the handlebody $\overline{M-N(\Gamma)}$ after it is cut along the properly embedded collection of punctured incompressible surfaces $2 \mathcal{F}^{\prime}=\overline{2 \mathcal{F}-N(\Gamma)}$. It is well known that when a handlebody is cut along a collection of incompressible surfaces, the resulting pieces are handlebodies. So $\overline{M^{\prime}-N(\Gamma)}$ is a handlebody and $\Gamma \cap M^{\prime}$ is the corresponding tunnel system.

The intersection of the 2-complex $\Gamma \cup \Delta$ with the incompressible surfaces $2 \mathcal{F}$ is a graph $G \subset 2 \mathcal{F}$. See Figure 7. A component of intersection with the spine, $\Gamma \cap 2 \mathcal{F}$ is called a vertex. Since handlebodies do not contain closed incompressible surfaces, there is at least one vertex in each component of $2 \mathcal{F}$. A component of the intersection with the compressing disks, $\Delta \cap 2 \mathcal{F}$ is called a circle if it is an intersection with the interior of $\Delta$ and an edge otherwise. An 
edge joining distinct vertices will be called an arc and an edge joining a vertex $v$ to itself is called a loop based at $v$.

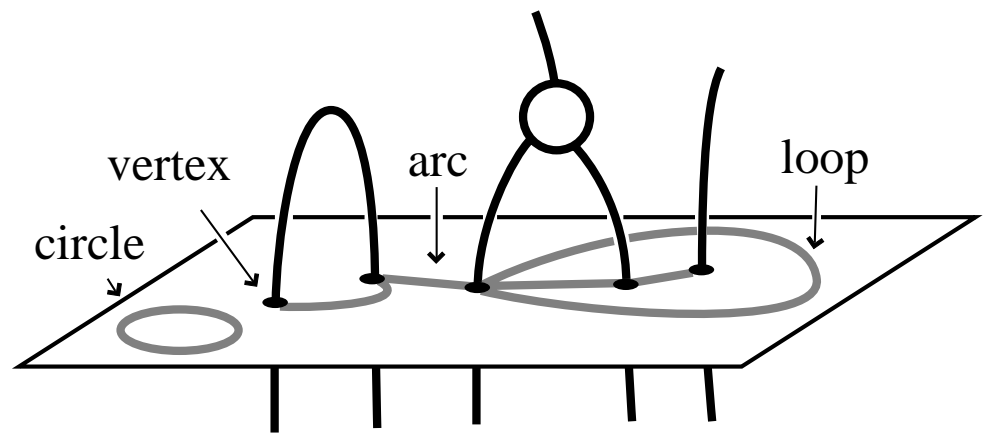

Figure 7: The intersection of $\Gamma \cup \Delta$ with $2 \mathcal{F}$ is a graph in $2 \mathcal{F}$.

\subsection{Lemma There are no circles in $G$.}

Proof This follows from the minimality of $\Delta \cap 2 \mathcal{F}$, using an innermost disk argument and Lemma 4.1.

4.4 Lemma [11] There are no isolated vertices (every vertex belongs to some edge).

Proof If some vertex is isolated then it defines a compressing disk $D$ for the handlebody $N(\Gamma)$ (or the vertex cuts off a tree, contradicting minimality). Moreover, the boundary of $D$ is disjoint from the complete collection of disks $\Delta$. After compressing the handlebody $\overline{M-N(\Gamma)}$ along $\Delta$ we obtain a collection of balls, and $\partial D$ is a loop on the boundary of one of these balls. It therefore also bounds a disk in the handlebody $\overline{M-N(\Gamma)}$. This implies that $\Gamma$ is the spine of a reducible Heegaard splitting.

We rely heavily on the notion of outermost edges [11]. Every edge $e$ of $G$ separates some disk $D \subset \Delta$ into two subdisks, $D_{1}$ and $D_{2}$. If one of the subdisks does not contain any other edges of $G$ then $e$ is called an outermost edge of $G$. Suppose that an edge $e$ is joined to the vertex $v$ and that one of the two subdisks $D_{1}$ or $D_{2}$ does not contain an edge of $G$ which is joined to $v$. Then, $e$ is an outermost edge with respect to $v$.

Note that by passing to subdisks, every vertex $v$ has some edge $e$ which is outermost with respect to it. Also, an outermost edge is outermost with respect to its vertices (or vertex), but not (in general) vice-versa. 
4.5 Lemma [11] Let $e$ be an outermost edge with respect to one of its vertices $v$. Then $e$ is a loop based at $v$ that is essential in $2 \mathcal{F}$.

Proof Suppose that $e$ is an arc and joins $v$ to a distinct vertex $w$. See Figure 8. (The edge $e$ may or may not be outermost for $w$.) The edge $e$ cuts off a disk $D^{\prime} \subset \Delta$ which does not contain any edge joined to $v$. Let $M^{\prime}$ be the adjacent manifold into which $D^{\prime}$ starts, and let $M^{\prime \prime}$ be adjacent manifold. Let $\gamma \subset \Gamma$ denote the handle containing $v$.

We will now perform a broken edge slide [11] which shows that the intersection is not minimal. See Figure 8. Add a new vertex to $\gamma$ that lies slightly into $M^{\prime}$, this breaks $\gamma$ into two handles, $\gamma_{1}$ and $\gamma_{2}$. Use the disk $D^{\prime}$ to guide an edge-slide of $\gamma_{1}$, which pulls it back into $M^{\prime \prime}$. This edge-slide is permissible precisely because $e$ is outermost for $v$, we did not ask the handle $\gamma_{1}$ to slide along itself. It does not increase the handle number of $\Gamma \cap M^{\prime \prime}$, while it strictly decreases the handle number of $\Gamma \cap M^{\prime}$ (and possibly others, if $\gamma_{2}$ runs through other manifolds). This contradicts the minimality of the intersection between $\Gamma$ and $2 \mathcal{F}$.
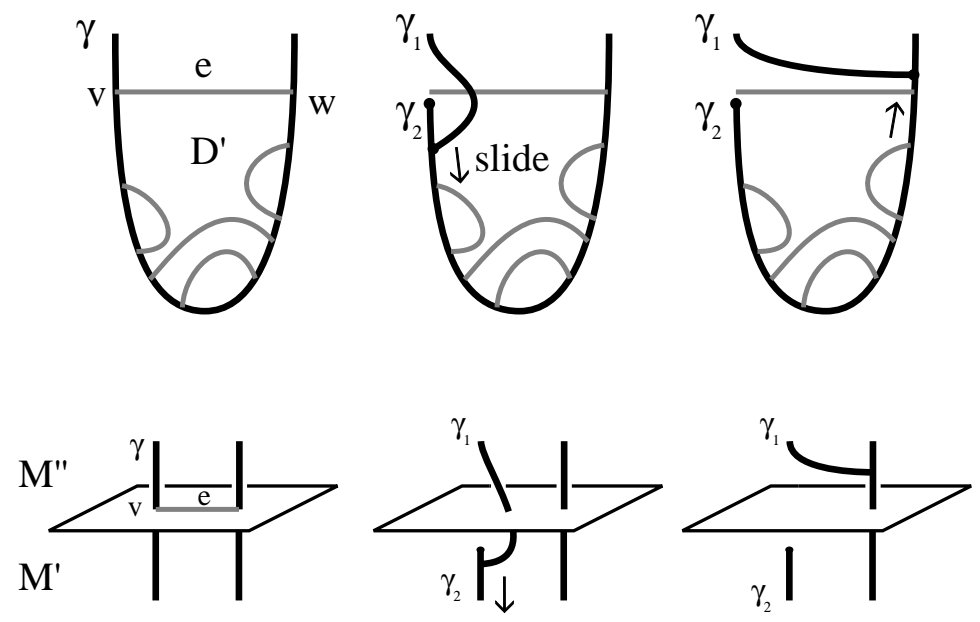

Figure 8: A broken edge slide.

We have established that an outermost edge for a vertex must be a loop. If it were inessential then we can find an innermost inessential loop bounding a disk $D$. If $D$ contains a vertex $v$ then an outermost edge for $v$ is an arc, contradicting the previous conclusion of this lemma. If $D$ does not contain a vertex, then we can reduce the number of intersections of $\Delta \cap 2 \mathcal{F}$ by boundary compressing $\Delta$ along $D$. See Figure 9 . 


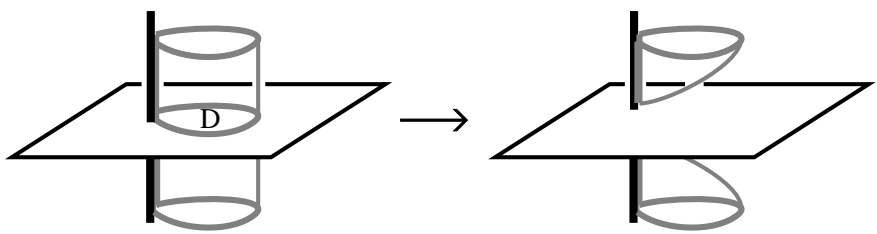

Figure 9: Boundary compressing $\Delta$ reduces the number of intersections.

4.6 Lemma The support of an outermost disk, supp $\left(d_{j}\right)$ is connected, has a single boundary vertex, and $h\left(\operatorname{supp}\left(d_{j}\right)\right)=g\left(\operatorname{supp}\left(d_{j}\right)\right)>0$.

Proof There is a single edge $e \subset G$ cutting off the outermost disk $d_{j}$ from $\Delta$. By Lemma 4.5, this edge is an essential loop in some component $F$ of $2 \mathcal{F}$. This implies that $\operatorname{supp}\left(d_{j}\right)$ has a single boundary vertex and is connected. Now, if $h\left(\operatorname{supp}\left(d_{j}\right)=0\right.$, then the subarc $\alpha=\partial d_{j}-e \subset \partial N(\Gamma)$ of the boundary of $d_{j}$ does not cross any compressing disk of $\Gamma \cap M_{i}$ other than the disk corresponding to the vertex. This means that we can perform edge slides of $\Gamma$ that allow us to pull the arc $\alpha$ back to $F$, creating an essential circle of intersection in the process. This is a contradiction, a subdisk of $d_{j}$ becomes a compressing disk for $F$, see Figure 10 .

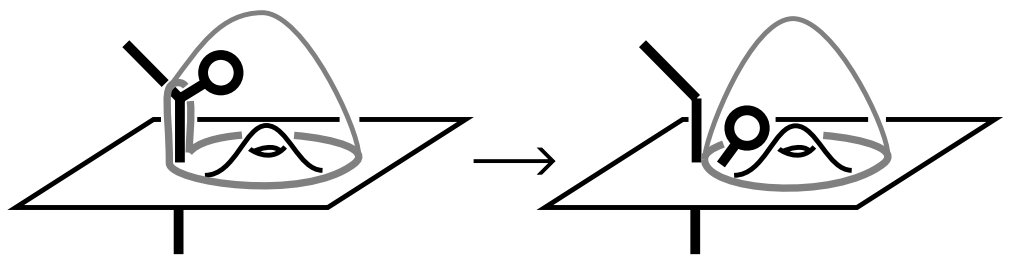

Figure 10: An outermost disk with handle number 0.

Since, $\operatorname{supp}\left(d_{j}\right)$ has a single boundary vertex, all of its handles must be realized by genus, i.e., $g\left(\operatorname{supp}\left(d_{j}\right)\right)=h\left(\operatorname{supp}\left(d_{j}\right)\right)$.

4.7 Lemma The support $\Omega_{i}$ of a relatively outermost disk $D_{i}$ for a component manifold $M_{i}$ is connected and has $h\left(\Omega_{i}\right) \geq 1$.

Proof We first show that $\Omega_{i}$ is connected. The boundary of the relatively outermost disk $D_{i}$ consists of arcs on $\Omega_{i}$ and edges lying in $2 \mathcal{F}$. Each arc in $\Omega_{i}$ lies in a single component of $\Omega_{i}$. All but at most one of the edges cuts off a disk which does not return to $M_{i}$. Each of these edges is therefore outermost 
for its vertices, and by Lemma 4.5 an essential loop in some component $F$ of $2 \mathcal{F}$. Loops do not join distinct components of $\Omega_{i}$. This means that $\Omega_{i}$ is connected, for any edge leaving a component there must be an additional edge that returns to that component, and we have at most one edge that is not a loop.

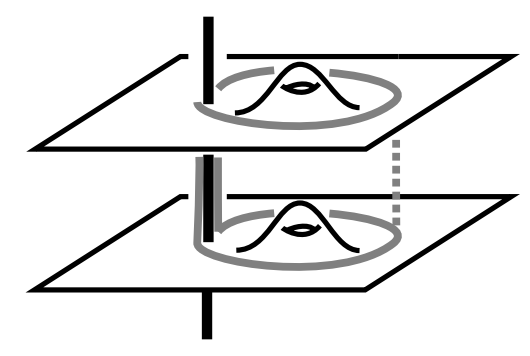

Figure 11: A relatively outermost disk that joins distinct boundary components.

Now, suppose that $h\left(\Omega_{i}\right)=0$. We know that all but at most one of the edges is a loop. While in general it is possible that the remaining edge $e$ is an edge, this does not occur when $h\left(\Omega_{i}\right)=0$. A single edge implies that the boundary of $D_{i}$ joins two distinct vertices in the graph and therefore traverses a handle in the component manifold.

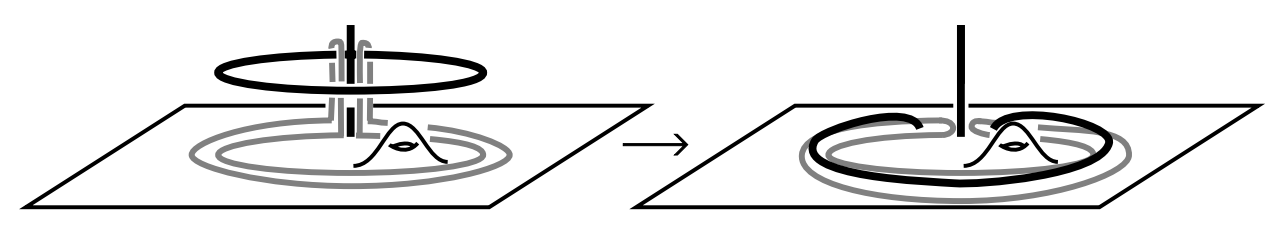

Figure 12: A relatively outermost disk whose support has handle number 0.

Since $h\left(\Omega_{i}\right)=0$ we may perform edge slides so that a sub disk of $D_{i}$ intersects some component $F$ of $2 \mathcal{F}$ in a circle that bounds a disk in $M_{i}$. See Figure 12 . This may raise the handle number of an adjacent product manifold. Since $F$ is incompressible, the boundary of this disk bounds a disk in $F$, the two disks bound a ball, and as in Lemma 4.1 we can perform an isotopy of the graph that reduces the induced handle number of the component manifold $M_{i}$. This contradicts minimality of the intersection of $\Gamma$ and $2 \mathcal{F}$.

There is one situation contradicting minimality that cannot be detected from the intersection of $\Gamma$ and $2 \mathcal{F}$ and the knowledge that an edge is outermost. It is possible that there is a loop based at a vertex $v$ that cuts off a disk 
lying in a component manifold which runs along a handle exactly once, see for example Figure 13. In this case, the handle can be slid into the product manifold reducing complexity. This is also the motivation for working with the collection $2 \mathcal{F}$ instead of $\mathcal{F}$ and choosing our definition of complexity. If we were working with a single copy, $\mathcal{F}$, this move would not decrease complexity, it raises the induced handle number of the adjacent component manifold. This situation will be detected by using the machinery of Casson and Gordon [1] and is analyzed in Section 6 .

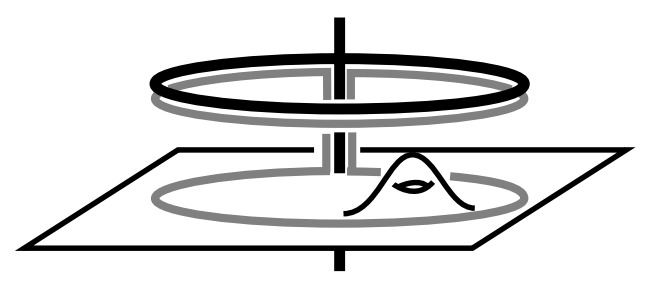

Figure 13: A handle that is parallel to a component of $\mathcal{F}$.

\section{Estimating the Genus of $\Gamma$}

The setup for this section is that of the proof of the main theorem: $\Gamma$ is the spine of an irreducible Heegaard splitting, $\Delta$ is a complete collection of compressing disk for $\overline{M-N(\Gamma)}$, both chosen to intersect $2 \mathcal{F}$ minimally; and $\Omega_{1}, \ldots, \Omega_{n}$ are the support of relatively outermost disks, $D_{1}, \ldots, D_{n}$, for the component manifolds, $M_{1}, \ldots, M_{n}$. We demonstrate that the sum of the handle numbers of the supports gives us a lower bound on the genus of $\Gamma$.

\subsection{Lemma}

$$
g(\Gamma) \geq \sum_{1}^{n} h\left(\Omega_{i}\right)-n+2
$$

Proof Recall that we have defined

$$
\Gamma_{k}=\operatorname{supp}\left(\left\{d_{i}\right\}_{i=1 . . k}\right),
$$

where $d_{1}, \ldots, d_{m}$ is an outermost ordering of the subdisks of $\Delta-2 \mathcal{F}$. The proof is an inductive one, demonstrating that when $\Gamma_{k} \subsetneq \Gamma$,

$$
g\left(\Gamma_{k}\right)-\left|\Gamma_{k}\right| \geq \sum_{\Omega_{i} \subset \Gamma_{k}}\left(h\left(\Omega_{i}\right)-1\right),
$$


where $\left|\Gamma_{k}\right|$ denotes the number of components of $\Gamma_{k}$. We then analyze the final attachment, when $\Gamma_{k}=\Gamma$. Note that at each stage we are attaching some portion of the spine $\Gamma$; the right hand side of the inequality can only increase when this portion is actually the support of the relatively outermost disk $\Omega_{i}$ for some component manifold $M_{i}$.

Let $k=1$. The graph $\Gamma_{1}$ is the support of the outermost disk $d_{1}$ which is embedded in either a component or product manifold $M^{\prime}$. By Lemma 4.6, $\Gamma_{1}$ is connected, has a single boundary vertex, and has positive genus. This means that $g\left(\Gamma_{1}\right)=h\left(\Gamma_{1}\right)$. If $M^{\prime}$ is a component manifold we have $g\left(\Gamma_{1}\right)-1 \geq$ $h\left(\Omega_{1}\right)-1$ and if $M^{\prime}$ is a product manifold we have $g\left(\Gamma_{1}\right)-1 \geq 0$. This establishes Inequality 1 for $k=1$.

Now, suppose that $k>1, \Gamma_{k} \subsetneq \Gamma$, and that $\Gamma_{k-1}$ satisfies the inductive hypothesis. If $d_{k}$ is not an relatively outermost disk for a component manifold, then we merely need to observe that the left-hand side of Inequality 1 does not decrease when we attach $\operatorname{supp}\left(d_{k}\right)$. It will decrease only if the number of components increases, which means that some component of $\operatorname{supp}\left(d_{k}\right)$ is not attached to $\Gamma_{k-1}$. But, this happens only if $d_{k}$ is an outermost disk, in which case $\operatorname{supp}\left(d_{k}\right)$ has a single component and there is an increase of genus to compensate for the additional component.

We are left in the case that $d_{k}$ is a relatively outermost disk $D_{i}$ for some component manifold $M_{i}$, then $\operatorname{supp}\left(d_{k}\right)=\Omega_{i}$. By Lemma 4.7, $\Omega_{i}$ is connected. Again, if $\Omega_{i}$ is not actually attached to $\Gamma_{k-1}$, an additional component is added, but then $d_{k}$ is actually an outermost disk, $\operatorname{supp}\left(d_{k}\right)$ is connected, has positive genus, and $h\left(\Omega_{i}\right)-1=g\left(\Omega_{i}\right)-1$ is added to both sides.

If $d_{k}$ is a relatively outermost disk $D_{i}$ for $M_{i}$, but not absolutely outermost (for example that in Figure 11), then all but at most one boundary vertex of $\Omega_{i}$ is attached to $\Gamma_{k-1}$. As noted in the proof of Lemma 4.7, all but at most one of the vertices of $\Omega_{i}$, has an outermost loop in $G$ attached to it that cuts off a subdisk of $\Delta$ containing only disks with labels $d_{i}$, where $i<k$. Each such edge of the disk is attached to a disk with strictly smaller labels, so all but one boundary vertex is attached to $\Gamma_{k-1}$.

So, for all but the first vertex attached, each attached vertex adds to the genus by one or reduces the number of components by 1 , see Figure 14. Moreover, any genus of $\Omega_{i}$ is added to the genus of $\Gamma_{k-1}$. We have added $g\left(\Omega_{i}\right)+\left|\partial \Omega_{i}\right|-2$ to the left-hand side of 1 , this is the same as $h\left(\Omega_{i}\right)-1$, which is added to the right side.

A similar analysis pertains for the final attachment, when $\Gamma_{k}=\Gamma$. However, in this case every vertex of $\operatorname{supp}\left(d_{k}\right)$ is attached to $\Gamma_{k-1}$, for there can be no 


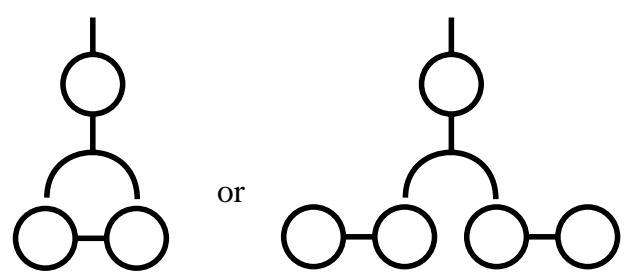

Figure 14: Attaching $\Omega_{i}$, a relatively outermost graph with handle number 3 , adds 2 to $g\left(\Gamma_{k-1}\right)-|\Gamma|$.
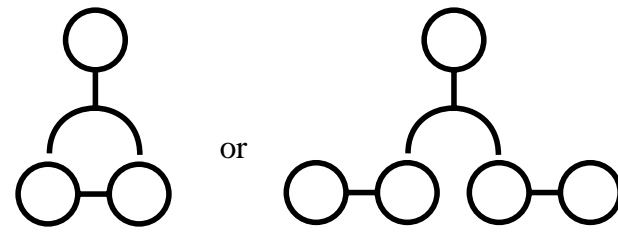

Figure 15: The final attachment.

unattached vertices, see Figure 15. When $\operatorname{supp}\left(d_{k}\right)$ is not some $\Omega_{i}$ this adds at least 1 to left side of Inequality 1 and nothing to the right hand side. When $\operatorname{supp}\left(d_{k}\right)=\Omega_{i}$ for some $i$, this adds $h\left(\Omega_{i}\right)$ to the left hand side and $h\left(\Omega_{i}\right)-1$ to the right side. In either case, the inequality will still hold even if we add an additional 1 to the right side. This yields

$$
g(\Gamma)-|\Gamma| \geq \sum_{1}^{n}\left(h\left(\Omega_{i}\right)-1\right)+1 .
$$

Since $\Gamma$ is connected, we have

$$
g(\Gamma) \geq \sum_{1}^{n} h\left(\Omega_{i}\right)-n+2 .
$$

\section{Weakly Reducible Heegaard splittings of Mani- folds with Boundary}

In [1] Casson and Gordon introduced the notion of a weakly reducible Heegaard splitting of a closed 3-manifold, and showed that such a splitting is either reducible or the manifold contains an incompressible surface. We first state and prove an extension of their theorem to manifolds with boundary; a similar theorem was proven by Lustig and Moriah [9]. We will then apply these techniques 
to the Heegaard splittings of the component manifolds $M_{i}$ that are induced by the Heegaard spine $\Gamma$. These splittings are typically weakly reducible.

First, we introduce some notation. Suppose that $H \subset M$ is a closed embedded surface and $\Delta \subset M$ is an embedded collection of disks so that $\Delta \cap H=\partial \Delta$. Let $\sigma(H, D)$ denote the surface obtained by performing an ambient 1-surgery of $H$ along $D$ (i.e., compression). We use the notion of complexity introduced in [1], the complexity of a surface is defined to be,

$$
c(\text { surface })=\sum(1-\chi(S)),
$$

where the sum is taken over all non-sphere components of the surface $S$. Note that if $D$ is a single disk with essential boundary then

$$
c(\sigma(H, D))= \begin{cases}c(H)-1 & \text { if } D \text { is separating or compresses a torus } \\ & \text { component of } H, \text { and } \\ c(H)-2 & \text { otherwise }\end{cases}
$$

6.1 Theorem Let $M$ be a compact, orientable, irreducible 3-manifold and $M=C_{1} \cup_{H} C_{2}$ a Heegaard splitting of $M$. If $\Delta_{0}=\Delta_{1} \cup \Delta_{2}$ is a weak reducing system for the Heegaard splitting then either

(1) $M$ contains an orientable, non-peripheral incompressible surface $S$ so that $c(S)$ is less than or equal to the complexity of $\sigma\left(H, \Delta_{0}\right)$, or

(2) there is an embedded collection of disks $\widehat{\Delta_{1}} \subset C_{1}$ so that $\Delta_{1} \subset \widehat{\Delta_{1}}$ and some component of $\sigma\left(H, \widehat{\Delta_{1}}\right)$ is a Heegaard surface for $M$, or

(3) there is an embedded collection of disks $\widehat{\Delta_{2}} \subset C_{2}$ so that $\Delta_{2} \subset \widehat{\Delta_{2}}$ and some component of $\sigma\left(H, \widehat{\Delta_{2}}\right)$ is a Heegaard surface for $M$.

In particular, conclusions (2) and (3) imply that $H$ is not of minimal genus.

Proof Define the complexity of a weak reducing system $\Delta_{0}=\Delta_{1} \cup \Delta_{2}$ for the Heegaard splitting $C_{1} \cup_{H} C_{2}$ to be

$$
c\left(\Delta_{0}\right)=c\left(\sigma\left(H, \Delta_{0}\right)\right) .
$$

Let the surfaces $H_{i}=\sigma\left(H, \Delta_{i}\right), i=0,1,2$, be obtained by compressing $H$ along the corresponding disk collections. See the schematic in Figure 16, it is essential to understanding the arguments of this section. Note that the surface $H_{0}$ separates $M$ into two components, denote these by $X_{1}$ and $X_{2}$. If we compress the compression body $C_{1}$ along the disk system $\Delta_{1}$ we obtain a compression body $Y_{1}$ which we will think of as sitting slightly inside $X_{1}$. Its 
complement $X_{1}-Y_{1}$ can be thought of as $\left(H_{1} \times I\right) \cup N\left(\Delta_{2}\right)$ - a product with 2-handles attached, and is therefore a compression body. Symmetrically, we also have that $X_{2}-Y_{2}$ is a compression body. Thus, the surfaces $H_{1}=\partial Y_{1}$ and $H_{2}=\partial Y_{2}$ are Heegaard surfaces for the (possibly disconnected) manifolds $X_{1}$ and $X_{2}$, respectively.
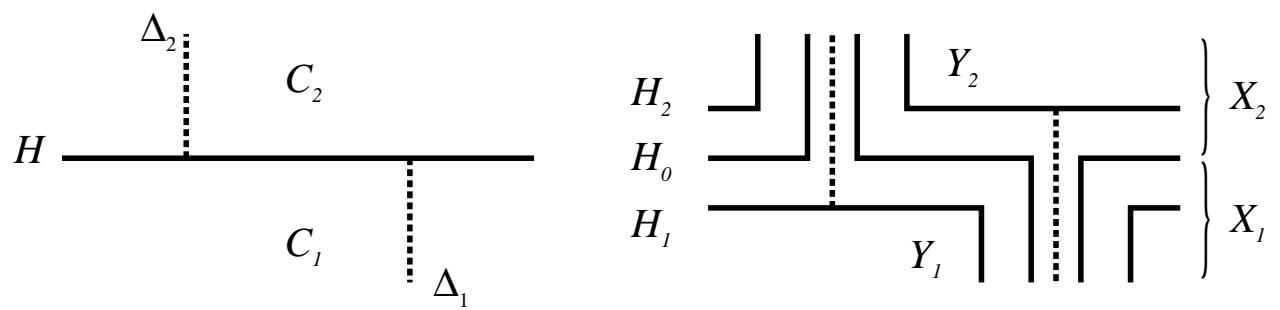

Figure 16: Compressing a Heegaard surface along a weak reducing system.

Suppose that some positive genus component of $H_{0}$ is compressible, say into $X_{1}$. The compressing disk $D$ for $H_{0}$ is a boundary reducing disk for the manifold $X_{1}$. As $H_{1}$ is a Heegaard surface for $X_{1}$, the Haken lemma (see [1]) implies that we may isotope $D$ to intersect $H_{1}$ in a single circle. It also says that we may choose a new collection of compressing disks $\Delta_{2}^{\prime}$ for $X_{1}-Y_{1}$, hence for $C_{2}$, which is disjoint from $D$. The collection $\Delta_{0}^{\prime}=\left(\Delta_{1} \cup D\right) \cup \Delta_{2}^{\prime}$ is a weak reducing system with lower complexity than $\Delta_{0}$ because we have compressed $H$ along an additional disk. A symmetric phenomenon occurs if $H_{0}$ is compressible into $X_{2}$.

In fact, we may continue to compress $H_{0}$, finding new disk collections of strictly decreasing complexity, until each component of $H_{0}$ is a 2-sphere or incompressible surface. Denote the final weak reducing system by $\Delta_{0}^{\prime}$, and the corresponding surfaces and sub-manifolds indicated in Figure 16 by $H_{1}^{\prime}, X_{1}^{\prime}, Y_{1}^{\prime}, \ldots$, etc.. Now $\Delta_{0}^{\prime}$ may or may not contain the original disk collections $\Delta_{1}$ and $\Delta_{2}$. However, the compression bodies $Y_{1}^{\prime}$ and $Y_{2}^{\prime}$ are obtained by compressing the compression bodies $Y_{1}$ and $Y_{2}$. These in turn were obtained by compressing $C_{1}$ and $C_{2}$ along the original collections $\Delta_{1}$ and $\Delta_{2}$. So we may also think of $Y_{1}^{\prime}$ and $Y_{2}^{\prime}$ as being obtained by compressing $C_{1}$ and $C_{2}$ along a collection of disks $\widehat{\Delta_{1}} \subset C_{1}$ and $\widehat{\Delta_{2}} \subset C_{2}$ where $\Delta_{1} \subset \widehat{\Delta_{1}}$ and $\Delta_{2} \subset \widehat{\Delta_{2}}$. In general $\widehat{\Delta_{1}}$ and $\widehat{\Delta_{2}}$ do not have disjoint boundary on $H$ and cannot be taken to be part of a weak reducing system.

If some component $S$ of $H_{0}^{\prime}$ is an incompressible and non-peripheral surface, then we have conclusion (1) of the theorem. Moreover, we have that $c(S) \leq$ 
$c\left(\sigma\left(H, \Delta_{0}\right)\right)$, for $S$ is a component of $H_{0}^{\prime}$ which was obtained by compressing $H_{0}=c\left(\sigma\left(H, \Delta_{0}\right)\right)$.

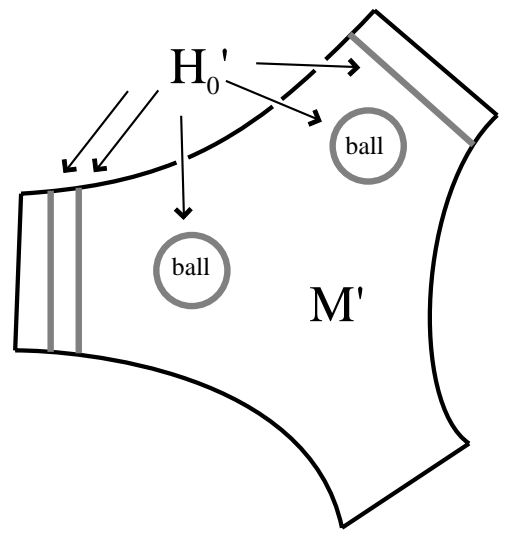

Figure 17: A component $M^{\prime}$ is essentially the same as $M$.

We therefore assume that each component of $H_{0}^{\prime}$ is a 2 -sphere bounding a ball ( $M$ is irreducible) or a peripheral incompressible surface. See Figure 17. Then some component of $M-N\left(H_{0}^{\prime}\right)$, call it $M^{\prime}$, is a copy of $M$ perhaps with some balls and product neighborhoods of boundary components of $M$ removed. Since $H_{0}^{\prime}$ separates $M$ into $X_{1}^{\prime}$ and $X_{2}^{\prime}, M^{\prime}$ must actually be a component of either $X_{1}^{\prime}$ or $X_{2}^{\prime}$, say $X_{1}^{\prime}$. Recall that the surface $H_{1}^{\prime}$ is a Heegaard surface for $X_{1}^{\prime}$. This means that some component $H^{\prime \prime} \subset H_{1}^{\prime}$ is a Heegaard surface for $M^{\prime}$. In fact, $H^{\prime \prime}$ is also a Heegaard surface for $M$; filling in balls and product neighborhoods of the appropriate boundary components does not change the property that $H^{\prime \prime}$ bounds compression bodies to both sides. Moreover, the Heegaard surface $H^{\prime \prime}$ is a component of the boundary of $Y_{1}^{\prime}$ and it follows from our earlier remarks, that it is a component of the surface $\sigma\left(H, \widehat{\Delta_{1}}\right)$, where $\Delta_{1} \subset \widehat{\Delta_{1}}$. Symmetrically, if $M^{\prime} \subset X_{2}^{\prime}$ then the Heegaard surface $H^{\prime \prime}$ is a component of the surface $\sigma\left(H, \widehat{\Delta_{2}}\right)$, where $\Delta_{2} \subset \widehat{\Delta_{2}}$.

There is one major difference between the case of closed manifolds addressed by Casson and Gordon and the case of bounded manifolds addressed in Theorem 6.1. Conclusions (2) and (3) in the above theorem do not imply that the splitting is reducible. A Heegaard splitting defines a partition of the boundary components of the manifold. Reducing (destabilizing) a Heegaard splitting does not change this partition of the boundary components, whereas compression along $\widehat{\Delta_{1}}$ or $\widehat{\Delta_{2}}$ may change the partition. This is seen in the following example. 
6.2 Example Consider $M$, the exterior of the three component chain pictured in Figure 18. (The manifold $M$ may also be though of as $P \times S^{1}$, where $P$ is a pair of pants).

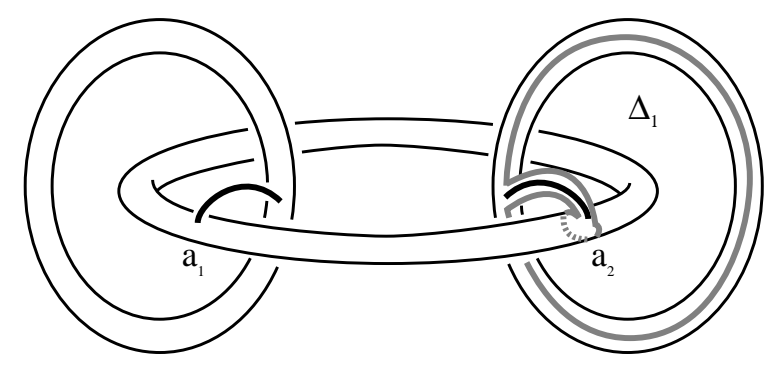

Figure 18: A weakly reducible tunnel system for the exterior of the three component chain.

It is not difficult to see that when a neighborhood of the arcs $a_{1}$ and $a_{2}$ are tunneled out of $M$, a handlebody is the result. Thus, $\left\{a_{1} \cup a_{2}\right\}$ is a tunnel system for $M$. Moreover, this system is weakly reducible: let $\Delta_{1}=D_{1}$ be the cocore of $a_{1}$ and $\Delta_{2}$ be the compressing disk for $M-N\left(a_{1} \cup a_{2}\right)$ whose boundary is indicated in the figure, running over $a_{2}$ twice. Since, $M$ does not contain any closed non-peripheral incompressible surfaces, Theorem 6.1 implies that this splitting can be compressed to a splitting of lower genus. However, the tunnel system $\left\{a_{1} \cup a_{2}\right\}$ cannot possibly be reducible, three is the minimal genus of a Heegaard splitting for which all three boundary components of $M$ are contained in the same compression body. In fact, either of the arcs $a_{1}$ or $a_{2}$ taken alone are a handle system for $M$. This induces a genus 2 Heegaard splitting of $M$ where one compression body contains two boundary components of $M$ and the other compression body contains one boundary component of $M$.

We now refine these methods to address the problem outlined in Section 3. The setup is the same as in that section: $M$ is a closed manifold, $\mathcal{F}$ is a complete collection of surfaces, $\Gamma$ is the spine of a Heegaard splitting that has been arranged to intersect $2 \mathcal{F}$ minimally, and $\Omega_{i}$ is the support of a relatively outermost disk $D_{i}$ for some component manifold $M_{i}$. The proof uses the notation and closely follows the proof of Theorem 6.1.

6.3 Proposition If $h\left(\Omega_{i}\right)=1$ then $\Omega_{i}$ is a handle system for $M_{i}$. In particular, $h\left(M_{i}\right) \leq 1$.

Proof By Theorem 4.2 we know that $\Gamma_{i}$ is a tunnel system for $M_{i} ; H=$ $\partial N\left(\Gamma_{i} \cup \partial M_{i}\right)$ is a Heegaard surface for $M_{i}$. We may write $M_{i}=C_{1} \cup_{H} C_{2}$ 
where $C_{1}$ is a compression body containing all components of $\partial M_{i}$ and $C_{2}$ is a handlebody.

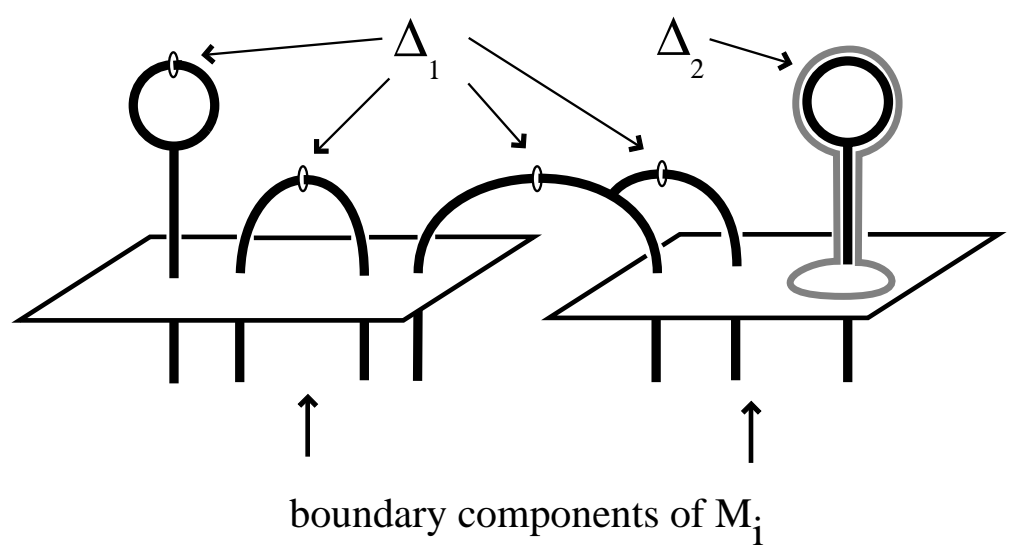

Figure 19: The tunnel system $\Gamma \cap M_{i}$ is weakly reducible.

If $h\left(\Gamma_{i}\right)=1$ then $\Omega_{i}=\Gamma_{i}$ and the result holds trivially. So we assume that $h\left(\Gamma_{i}\right)>1$. This implies that $M_{i}=C_{1} \cup_{H} C_{2}$ is weakly reducible: let $\Delta_{1} \subset C_{1}$ be a complete collection of compressing disks for $N\left(\Gamma_{i}-\Omega_{i}\right)$, and $\Delta_{2}=D_{i}$, the relatively outermost disk. See Figure 19. We may choose $\Delta_{1}$ so that $Y_{1}$ does not contain any balls, every component is attached to $\partial M$.

Following the proof of Theorem 6.1, by further compressing $H_{0}$ we obtain a sequence of weak reducing systems, with strictly decreasing complexity. Let $\Delta_{0}^{\prime}=\Delta_{1}^{\prime} \cup \Delta_{2}^{\prime}$ be the final disk system; for this system the surface $H_{0}^{\prime}$ consists of 2-spheres and incompressible surfaces.

Claim Every incompressible component of $H_{0}^{\prime}$ is peripheral.

Otherwise, by Theorem 6.1 $M_{i}$ contains an orientable, non-peripheral incompressible surface $S$. Recall that $H_{1}$ is the surface obtained by compressing $H$ along $\Delta_{1}$, in this case it consists of copies of boundary components (possibly none), and either one or two boundary components with a handle, $\partial N\left(\Omega_{i}\right)$, attached. To obtain $S$, we further compress along $\Delta_{2}=D_{i}$ and perhaps along additional disks. Therefore $S$ has genus less than or equal to the genus of a boundary component when $\Omega_{i}$ is attached to a single boundary component, or strictly less than the sum of the genera of two boundary components when $\Omega_{i}$ is attached to two boundary components. This violates our assumption that the decomposition along $\mathcal{F}$ was complete. This completes the proof of the claim. 
We conclude, as in Theorem 6.1, that some component $M^{\prime} \subset X_{1}^{\prime}$ or $M^{\prime} \subset X_{2}^{\prime}$ is a copy of $M_{i}$ with some balls removed.

Claim If $M^{\prime} \subset X_{1}^{\prime}$ then $\Omega_{i}$ is a handle system for $M_{i}$.

In this case some component of $H_{1}^{\prime}=\partial Y_{1}^{\prime}$ is a Heegaard surface for $M_{i}$. Recall that $Y_{1}^{\prime}$ was obtained by first compressing $C_{1}=N\left(\partial M_{i} \cup \Gamma_{i}\right)$ along $\Delta_{1}$ yielding $Y_{1}=\left(\partial M_{i} \times I\right) \cup N\left(\Omega_{i}\right)$, and then perhaps compressing further. But, the only compressions remaining are along the cocore of $N\left(\Omega_{i}\right)$ or the separating disk which is the double of the cocore (only if $\Omega_{i}$ is attached to a single component of $\left.\partial M_{i}\right)$. But, we could not have compressed along either of these: compressing along the cocore leaves $Y_{1}^{\prime}=\partial M_{i} \times I$ whose boundary cannot include a Heegaard surface ( $M_{i}$ is not a compression body), and compressing along the double would imply that the Heegaard surface is the boundary of a solid torus, in particular $M_{i}$ has genus 1 . This is not possible since $\partial M_{i}$ has positive genus. Thus $\Delta_{0}$ is the final weak reducing system and $H_{1}^{\prime}=H_{1}$. The Heegaard surface is the boundary of the component of $Y_{1}$ that includes $N\left(\Omega_{i}\right)$. In other words, $\Omega_{i}$ is a handle system for $M_{i}$. (Recall Figure 16). This completes the claim.

The theorem will follow from the proof of the following claim. In it we argue that in fact, the initial disk system $\Delta_{0}$ is "almost" the final system $\Delta_{0}^{\prime}$. There may be one additional compression, but, it can be controlled.

Claim If $M^{\prime} \subset X_{2}^{\prime}$ then the intersection of $\Gamma$ with $2 \mathcal{F}$ is not minimal (a contradiction).

Since $M^{\prime}$ is isotopic to $M$ (modulo balls), for each boundary component, $M^{\prime}$ either contains that boundary component or a parallel copy of that boundary component. In fact, each must be a parallel copy: the original Heegaard surface $H$ separated the handlebody $C_{2}$ from the boundary components, and then so must $H_{0}^{\prime}$ separate $X_{2}^{\prime}$ from the boundary components. (Figure 16).

Since $M^{\prime} \subset X_{2}^{\prime}$ we have that $H_{0}^{\prime}=\partial X_{2}^{\prime}$ contains at least one parallel copy of each boundary component of $M_{i}$. It follows that

$$
c\left(H_{0}^{\prime}\right) \geq c\left(\partial M_{i}\right) .
$$

We now show that the surface $H_{0}$ contains a parallel copy of each component of $\partial M_{i}$. Denote the component(s) to which the handle $\Omega_{i}$ is attached by $\partial_{1} M_{i}$, and the others by $\partial_{2} M_{i}$. The surface $H_{1}$ was obtained by compressing along 
$\Delta_{1}$ and therefore contains a copy of each component of $\partial_{1} M_{i}$ and a copy of $\partial N\left(\Omega_{i}\right) \cup \partial_{2} M_{i}$. It follows that

$$
c\left(H_{1}\right)= \begin{cases}c\left(\partial M_{i}\right)+2 & \text { if }\left|\partial_{1} M_{i}\right|=1, \\ c\left(\partial M_{i}\right)+1 & \text { if }\left|\partial_{1} M_{i}\right|=2 .\end{cases}
$$

The complexity of $H_{0}$ is less by one if $\Delta_{2}=D_{i}$ separates and less by two if $\Delta_{2}=D_{i}$ does not separate. Unless $\Omega_{i}$ is attached to a single component and $D_{i}$ separates, we have $c\left(H_{0}\right) \leq c\left(\partial M_{i}\right)$. But we know that $c(\partial M) \leq c\left(H_{0}^{\prime}\right) \leq$ $c\left(H_{0}\right)$. This implies that $H_{0}=H_{0}^{\prime}$ and by previous comments contains a copy of each boundary component.

The remaining case is that $\partial_{1} M_{i}$ is a single component and $\Delta_{2}$ is separating. In this case $H_{0}$ contains $\partial_{2} M_{i}$ and two other components, $S^{\prime}$ and $S^{\prime \prime}$, each with positive genus. In particular, $c\left(H_{0}\right)=c\left(\partial M_{i}\right)+1$. There can only be one additional compression, say to $S^{\prime}$. But this implies that $S^{\prime \prime}$ is incompressible and hence is a copy of $\partial_{1} M_{i}$. In this case $S^{\prime \prime}$ is a compressible torus.

We now know that the surface $H_{0}$ is a single parallel copy of each boundary component and possibly a single compressible torus (see the proof of the above claim). The disks $\Delta_{1}$ were chosen so that $C_{1}$, and hence $X_{1}$, contains at most one component for each component of $\partial M_{i}$. Therefore the torus, if it exists, is contained in a product neighborhood of the boundary and some component of $X_{2}$ is a manifold $M^{\prime}$ isotopic to $M$. See Figure 20.

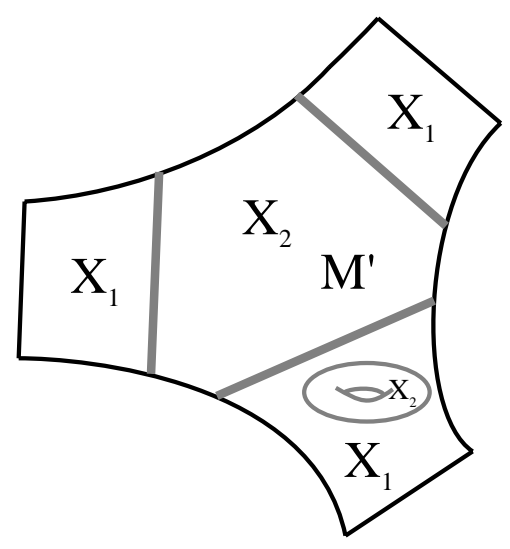

Figure 20: A component of $X_{2}$ is isotopic to $M$.

How does the spine $\Gamma$ intersect $M^{\prime}$ ? As $M^{\prime}$ is a component of $X_{2}$ it is formed by attaching the 2-handles $N\left(\Delta_{1}\right)$ to a handlebody $C_{2}$ which is disjoint from $\Gamma$, again Figure 16. But $\Gamma$ intersects $X_{2}$ in a single arc for each disk in $\Delta_{1}$ and the 
number of these is strictly less than $h\left(\Gamma \cap M_{i}\right)$. Then $\Gamma$ intersects $M^{\prime} \subset X_{2}^{\prime}$ in a subset of these arcs (perhaps all). By shrinking $M_{i}$ to $M^{\prime}$ we reduce $h\left(\Gamma \cap M_{i}\right)$ to $\left|\Delta_{1}\right|$ which is less by at least one (the handle $\Omega_{i}$ ). This contradicts the fact that $\sum_{1}^{n} h\left(\Gamma \cap M_{i}\right)$ was chosen to be minimal. Note that this may increase the handle number of the intersection of $\Gamma$ with the product manifold adjacent to $M_{i}$. Also note that the situation in this claim is precisely the situation that occurs when the outermost disk demonstrates that its support is parallel to the boundary surface, recall Figure 13. This completes the proof of the claim and the theorem.

Remark It is in the last claim of the proof that we are using the fact that $h\left(\Omega_{i}\right)=1$. It works because we have one handle, $\Omega_{i}$, and one compressing disk for the complement, $\Delta_{2}=D_{i}$. This implies that the original disk system $\Delta_{0}$ is "almost" the final compressing system $\Delta_{0}^{\prime}$. If the handle number were greater than one then we would have a discrepancy between the handle number of $\Omega_{i}$ and the number of compressions in $\Delta_{2}$, we would need to compress $C_{2}$ further, and be forced to change from the original disk collection $\Delta_{1}$ to a new disk collection $\Delta_{1}^{\prime}$. We cannot directly conclude that $\Gamma$ intersects each disk of $\Delta_{1}^{\prime}$ exactly once and the intersection of $\Gamma$ with $M^{\prime}$ might not be lower than that with $M_{i}$.

\section{References}

[1] A. Casson and C. Gordon. Reducing Heegaard splittings. Topology and its Applications, 27:275 - 283, 1987.

[2] M. Eudave-Muñoz. Incompressible surfaces in tunnel number one knots complements. Preprint.

[3] Charles Frohman. The topological uniqueness of triply periodic minimal surfaces in $\mathbb{R}^{3}$. J. Differential Geom., 31(1):277-283, 1990.

[4] Wolfgang Haken. Some results on surfaces in 3-manifolds. In Studies in Modern Topology, pages 39-98. Math. Assoc. Amer. (distributed by Prentice-Hall, Englewood Cliffs, N.J.), 1968.

[5] William Jaco. Lectures on three-manifold topology. American Mathematical Society, Providence, R.I., 1980.

[6] Klaus Johannson. Topology and combinatorics of 3-manifolds. Springer-Verlag, Berlin, 1995.

[7] Tsuyoshi Kobayashi. Structures of the Haken manifolds with Heegaard splittings of genus two. Osaka J. Math., 21(2):437-455, 1984. 
[8] Tsuyoshi Kobayashi. A construction of arbitrarily high degeneration of tunnel numbers of knots under connected sum. J. Knot Theory Ramifications, 3(2):179186,1994 .

[9] M. Lustig and Y. Moriah. Closed incompressible surfaces in complements of wide knots and links. Topology and its Applications, 92:1 - 13, 1992.

[10] M. Scharlemann. Hegaard splittings of compact 3-manifolds. To appear in Handbook of Geometric Topology, ed by R. Daverman and R. Sherr, Elsevier Press.

[11] M. Scharlemann and A. Thompson. Thin position and Heegaard splittings of the 3-sphere. J. Diff. Geom., 39(2):343-357, 1994.

[12] M. Scharlemann and A. Thompson. Thin position for 3-manifolds. In Geometric topology (Haifa, 1992), pages 231-238. Amer. Math. Soc., Providence, RI, 1994.

[13] J. Schultens. Additivity of tunnel number for small knots. Comment. Math. Helv., 75(3): 353-367, 2000.

DePaul University, Department of Computer Science 243 S Wabash Ave, Chicago, IL 60604, USA

Email: esedgwick@cs.depaul.edu

Received: 18 April 2001 Revised: 27 July 2001 